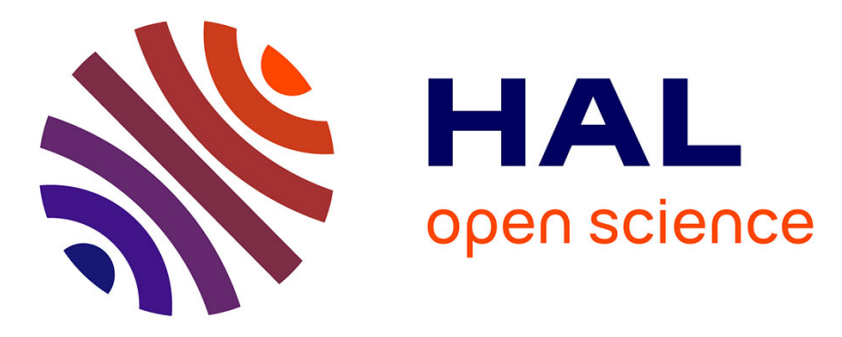

\title{
Efficient On-the-fly Algorithms for the Analysis of Timed Games
}

Franck Cassez, Alexandre David, Emmanuel Fleury, Kim Guldstrand Larsen, Didier Lime

\section{- To cite this version:}

Franck Cassez, Alexandre David, Emmanuel Fleury, Kim Guldstrand Larsen, Didier Lime. Efficient On-the-fly Algorithms for the Analysis of Timed Games. 16th International Conference on Concurrency Theory (CONCUR'05), 2005, San Francisco, United States. pp.66-80, 10.1007/11539452 . hal-00350475

\section{HAL Id: hal-00350475 \\ https://hal.science/hal-00350475}

Submitted on 6 Jan 2009

HAL is a multi-disciplinary open access archive for the deposit and dissemination of scientific research documents, whether they are published or not. The documents may come from teaching and research institutions in France or abroad, or from public or private research centers.
L'archive ouverte pluridisciplinaire HAL, est destinée au dépôt et à la diffusion de documents scientifiques de niveau recherche, publiés ou non, émanant des établissements d'enseignement et de recherche français ou étrangers, des laboratoires publics ou privés. 


\title{
Efficient On-the-fly Algorithms for the Analysis of Timed Games
}

\author{
Franck Cassez ${ }^{1}$, Alexandre David ${ }^{2}$, Emmanuel Fleury $^{2}$, Kim G. Larsen ${ }^{2}$, \\ Didier Lime ${ }^{2}$ \\ 1 IRCCyN, UMR 6597, CNRS, France \\ Franck.Cassez@irccyn.ec-nantes.fr \\ 2 Computer Science Department \\ CISS (Center for Embedded Software Systems), \\ Aalborg University, Denmark \\ \{adavid,fleury, kgl, didier\}@cs.aau.dk
}

\begin{abstract}
In this paper, we propose a first efficient on-the-fly algorithm for solving games based on timed game automata with respect to reachability and safety properties ${ }^{1}$.

The algorithm we propose is a symbolic extension of the on-the-fly algorithm suggested by Liu \& Smolka [15] for linear-time model-checking of finite-state systems. Being on-the-fly, the symbolic algorithm may terminate long before having explored the entire state-space. Also the individual steps of the algorithm are carried out efficiently by the use of so-called zones as the underlying data structure.

Various optimizations of the basic symbolic algorithm are proposed as well as methods for obtaining time-optimal winning strategies (for reachability games). Extensive evaluation of an experimental implementation of the algorithm yields very encouraging performance results.
\end{abstract}

\section{Introduction}

On-the-fly algorithms offer the benefit of settling properties of individual system states (e.g. an initial state) in a local fashion and without necessarily having to generate or examine the entire state-space of the given model. For finite-state (untimed) systems the search for optimal (linear) on-the-fly or local algorithms has been a very active research topic since the end of the 80 's $[12,4,15]$ and is one of the most important techniques applied in finite-state model-checkers using enumerative or explicit state-space representation, as is the case with SPIN [10], which performs on-the-fly model-checking of LTL properties.

Also for timed systems, on-the-fly algorithms have been absolutely crucial to the success of model-checking tools such as KronOs [8] and UPPAAL [13] in their analysis of timed automata based models [2]. Both reachability, safety as

${ }^{1}$ Though timed games for long have been known to be decidable there has until now been a lack of efficient and truly on-the-fly algorithms for their analysis. 
well as general liveness properties of such timed models may be decided using on-the-fly algorithms exploring the reachable state-space in a (symbolic) forward manner with the possibility of early termination. More recently, timed automata technology has been successfully applied to optimal scheduling problems with guiding and pruning heuristics being added to yield on-the-fly algorithms which quickly lead to near-optimal (time- or cost-wise) schedules $[5,3,11,18]$.

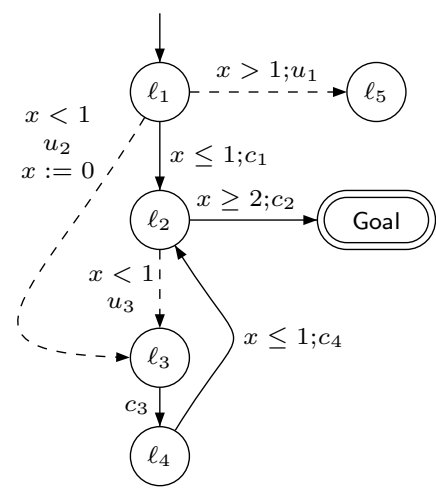

Fig. 1. A Timed Game Automaton

We consider timed game automata and how to decide the existence of a winning strategy w.r.t. reachability or safety. As an example, consider the timed game automaton $A$ of Fig. 1 consisting of a timed automaton with one clock $x$ and two types of edges: controllable $\left(c_{i}\right)$ and uncontrollable $\left(u_{i}\right)$. The reachability game consists in finding a strategy for a controller, i.e. when to take the controllable transitions that will guarantee that the system, regardless of when and if the opponent chooses to take uncontrollable transitions, will eventually end up in the location Goal. Obviously, for all initial states of the form $\left(\ell_{1}, x\right)$ with $x \leq 1$ there is such a winning strategy ${ }^{2}$.

Though such timed game automata for long have been known to be decidable $[16,6,9]$ there is still a lack of efficient and truly on-the-fly algorithms for their analysis. Most of the suggested algorithms are based on backwards fix-point computations of the set of winning states $[16,6,9]$. In contrast, the on-the-fly algorithms used for model-checking timed automata models (w.r.t. reachability) makes a forward symbolic state-space exploration resulting in the so-called simulation graph. However, the simulation graph is by itself too abstract to be used as the basis for an on-the-fly algorithm for computing winning strategies. Fig. 2 (a) gives the simulation graph of the timed game automata of Fig. 1, which incorrectly classifies the initial state as being uncontrollable when viewed as a finite-state game.

As a remedy to this problem, the authors of $[20,1]$ propose a partially onthe-fly method for solving reachability games for a timed game automaton $A$. However, this method involves an extremely expensive preprocessing step in which the quotient graph of the dense time transition system $S_{A}$ w.r.t. timeabstracted bisimulation ${ }^{3}$ needs to be built. Once obtained this quotient graph may be used with any on-the-fly game-solving algorithm for untimed (finitestate) systems. As an illustration, Fig. 2 (b) gives the time abstracted quotient

\footnotetext{
${ }^{2}$ A winning strategy would consist in taking $c_{1}$ immediately in all states $\left(\ell_{1}, x\right)$ with $x \leq 1$; taking $c_{2}$ immediately in all states $\left(\ell_{2}, x\right)$ with $x \geq 2$; taking $c_{3}$ immediately in all state $\left(\ell_{3}, x\right)$ and delaying in all states $\left(\ell_{4}, x\right)$ with $x<1$ until the value of $x$ is 1 at which point the edge $c_{4}$ is taken.

${ }^{3}$ A time-abstracted bisimulation is a binary relation on states preserving discrete states and abstracted delay-transitions.
} 

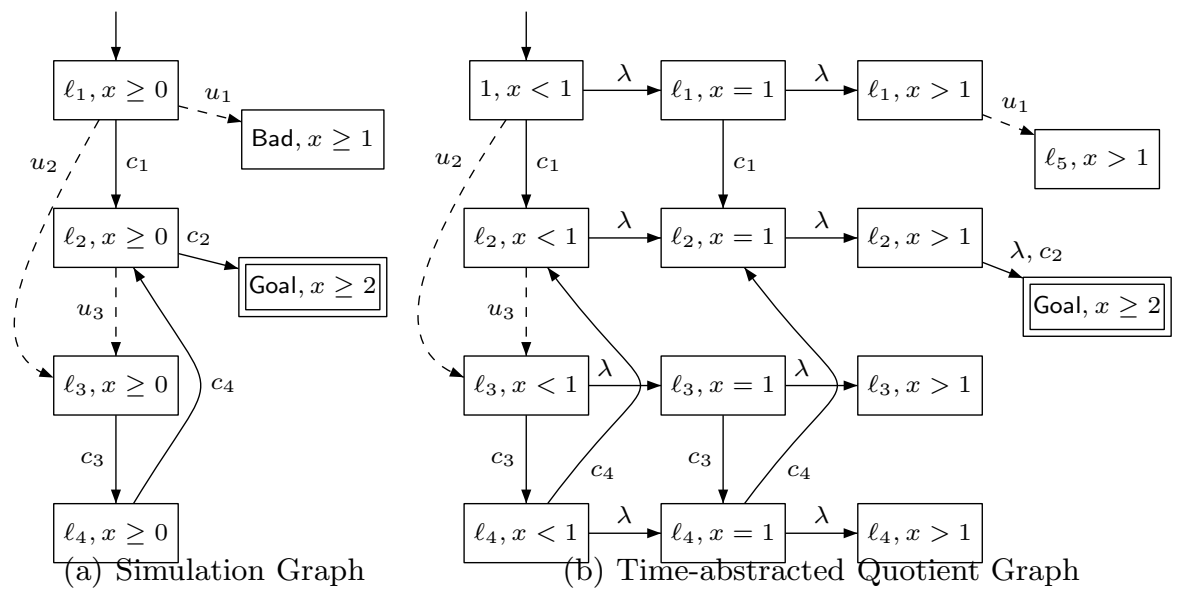

Fig. 2. Simulation and time-abstracted quotient graph of Fig. 1 ( $\lambda$ is for time elapsing)

graph for the timed game automaton of Fig. 1. It should be easy for the reader to see that the initial state will now (correctly) be classified as controllable.

In this paper, we propose an efficient, truly on-the-fly algorithm for the computation of winning states for timed game automata. Our algorithm is a symbolic extension of the on-the-fly algorithm suggested by Liu \& Smolka [15] for linear-time model-checking of finite-state systems. Being on-the-fly, the symbolic algorithm may terminate before having explored the entire state-space, i.e. as soon as a winning strategy has been identified. Also the individual steps of the algorithm are carried out efficiently by the use of so-called zones as the underlying data structure.

The rest of the paper is organized as follows. Section 2 provides definitions and preliminaries about timed game automata and classic backwards algorithm for solving them. Section 3 presents our instantiation of the general on-the-fly algorithm of Liu \& Smolka [15] to untimed reachability games. Then, in Section 4, we present our symbolic extension of this algorithm, providing a first forward, zone-based and fully on-the-fly algorithm for solving timed reachability games. Section 5 discusses few optimizations of the basic algorithm and how to apply the algorithm to determine time-optimal winning strategies. Section 6 presents experimental evaluation of an efficient implementation of the algorithm to determine (time-optimal) winning strategies. The performance results obtained are very encouraging. Finally, Section 7 presents conclusion and future work and all proofs can be found in the Appendix . 


\section{Backward Algorithms for Solving Timed Games}

Timed Game Automata [16] (TGA) were introduced for control problems on timed systems. This section recalls basic results of controller synthesis for TGA.

Let $X$ be a finite set of real-valued variables called clocks. We note $\mathcal{C}(X)$ the set of constraints $\varphi$ generated by the grammar: $\varphi::=x \sim k|x-y \sim k| \varphi \wedge \varphi$ where $k \in \mathbb{Z}, x, y \in X$ and $\sim \in\{<, \leq,=,>, \geq\} . \mathcal{B}(X)$ is the subset of $\mathcal{C}(X)$ that uses only rectangular constraints of the form $x \sim k$. A valuation of the variables in $X$ is a mapping $X \mapsto \mathbb{R}_{\geq 0}$ (thus $\mathbb{R}_{\geq 0}^{X}$ ). We write $\overrightarrow{0}$ for the valuation that assigns 0 to each clock. For $Y \subseteq X$, we denote by $v[Y]$ the valuation assigning $0($ resp. $v(x)$ ) for any $x \in Y$ (resp. $x \in X \backslash Y)$. We denote $v+\delta$ for $\delta \in \mathbb{R}_{\geq 0}$ the valuation s.t. for all $x \in X,(v+\delta)(x)=v(x)+\delta$. For $g \in \mathcal{C}(X)$ and $v \in \mathbb{R}_{>0}^{X}$, we write $v \models g$ if $v$ satisfies $g$ and $\llbracket g \rrbracket$ denotes the set of valuations $\left\{v \in \mathbb{R}_{\geq 0}^{X} \mid v \models g\right\}$. A zone $\mathrm{Z}$ is a subset of $\mathbb{R}_{\geq 0}^{X}$ s.t. $\llbracket g \rrbracket=Z$ for some $g \in \mathcal{C}(X)$.

\subsection{Timed Game Automata \& Simulation Graph}

Definition 1 (Timed Automaton [2]). A Timed Automaton (TA) is a tuple $A=\left(L, \ell_{0}\right.$, Act, $X, E$, Inv) where $L$ is a finite set of locations, $\ell_{0} \in L$ is the initial location, Act is the set of actions, $X$ is a finite set of real-valued clocks, $E \subseteq L \times \mathcal{B}(X) \times$ Act $\times 2^{X} \times L$ is a finite set of transitions, Inv : $L \rightarrow \mathcal{B}(X)$ associates to each location its invariant.

A state of a TA is a pair $(\ell, v) \in L \times \mathbb{R}_{\geq 0}^{X}$ that consists of a discrete part and a valuation of the clocks. From a state $(\ell, v) \in L \times \mathbb{R}_{>0}^{X}$ s.t. $v \models \operatorname{lnv}(\ell)$, a TA can either let time progress or do a discrete transition and reach a new state. This is defined by the transition relation $\longrightarrow$ built as follows: for $a \in$ Act, $(\ell, v) \stackrel{a}{\longrightarrow}\left(\ell^{\prime}, v^{\prime}\right)$ if there exists a transition $\ell \stackrel{g, a, Y}{\longrightarrow} \ell^{\prime}$ in $E$ s.t. $v \models g, v^{\prime}=v[Y]$ and $v^{\prime} \models \operatorname{lnv}\left(\ell^{\prime}\right)$; for $\delta \geq 0,(\ell, v) \stackrel{\delta}{\longrightarrow}\left(\ell, v^{\prime}\right)$ if $v^{\prime}=v+\delta$ and $v, v^{\prime} \in \llbracket \operatorname{lnv}(\ell) \rrbracket$. Thus the semantics of a TA is the labeled transition system $S_{A}=\left(Q, q_{0}, \longrightarrow\right)$ where $Q=L \times \mathbb{R}_{\geq 0}^{X}, q_{0}=\left(\ell_{0}, \overrightarrow{0}\right)$ and the set of labels is Act $\cup \mathbb{R}_{\geq 0}$. A run of a timed automaton $A$ is a sequence of alternating time and discrete transitions in $S_{A}$. We use $\operatorname{Runs}((\ell, v), A)$ for the set of runs that start in $(\ell, v)$. We write $\operatorname{Runs}(A)$ for $\operatorname{Runs}\left(\left(\ell_{0}, \overrightarrow{0}\right), A\right)$. If $\rho$ is a finite run we denote last $(\rho)$ the last state of the run and Duration $(\rho)$ the total elapsed time all along the run.

The analysis of TA is based on the exploration of a finite graph, the simulation graph, where the nodes are symbolic states; a symbolic state is a pair $(\ell, Z)$ where $\ell \in L$ and $Z$ is a zone of $\mathbb{R}_{\geq 0}^{X}$. Let $X \subseteq Q$ and $a \in$ Act we define the $a$-successor of $X$ by $\operatorname{Post}_{a}(X)=\left\{\left(\ell^{\prime}, v^{\prime}\right) \mid \exists(\ell, v) \in X,(\ell, v) \stackrel{a}{\longrightarrow}\left(\ell^{\prime}, v^{\prime}\right)\right\}$ and the $a$-predecessor $\operatorname{Pred}_{a}(X)=\left\{(\ell, v) \mid \exists\left(\ell^{\prime}, v^{\prime}\right) \in X,(\ell, v) \stackrel{a}{\longrightarrow}\left(\ell^{\prime}, v^{\prime}\right)\right\}$. The timed successors and predecessors of $X$ are respectively defined by $X^{\nearrow}=\{(\ell, v+d) \mid(\ell, v) \in X \cap$ $\left.\llbracket \operatorname{lnv}(\ell) \rrbracket,(\ell, v+d) \in \llbracket \operatorname{lnv}(\ell) \rrbracket, d \in \mathbb{R}_{\geq 0}\right\}$ and $X^{\swarrow}=\{(\ell, v-d) \mid(\ell, v) \in X, d \in$ $\left.\mathbb{R}_{>0}\right\}$. Let $\rightarrow$ be the relation defined on symbolic states by: $(\ell, Z) \stackrel{a}{\longrightarrow}\left(\ell^{\prime}, Z^{\prime}\right)$ if $\left(\ell, g, a, Y, \ell^{\prime}\right) \in E$ and $Z^{\prime}=((Z \cap \llbracket g \rrbracket)[Y])^{\nearrow}$. The simulation graph $S G(A)$ of $A$ is defined as the transition system $\left(Z(Q), S_{0}, \rightarrow\right)$, where $Z(Q)$ is the set of zones of $Q, S_{0}=\left(\left(\left\{\ell_{0}, \overrightarrow{0}\right\}\right\}^{\nearrow}\right) \cap \llbracket \operatorname{lnv}\left(\ell_{0}\right) \rrbracket$ and $\rightarrow$ defined as above. 
Definition 2 (Timed Game Automaton [16]). A Timed Game Automaton (TGA) $G$ is a timed automaton with its set of actions Act partitioned into controllable $\left(\mathrm{Act}_{c}\right)$ and uncontrollable $\left(\mathrm{Act}_{u}\right)$ actions.

\subsection{Safety and Reachability Games}

Given a TGA $G$ and a set of states $K \subseteq L \times \mathbb{R}_{>0}^{X}$ the reachability control problem consists in finding a strategy $f$ s.t. $G$ supervised by $f$ enforces $K$. The safety control problem is the dual asking for the strategy to constantly avoid $K$. By "a reachability game $(G, K)$ " (resp. safety) we refer to the reachability (resp. safety) control problem for $G$ and $K$.

Let $(G, K)$ be a reachability (resp. safety) game. A finite or infinite (ruling out runs with an infinite number of consecutive time transitions of duration 0 ) run $\rho=\left(\ell_{0}, v_{0}\right) \stackrel{e_{0}}{\longrightarrow}\left(\ell_{1}, v_{1}\right) \stackrel{e_{1}}{\longrightarrow} \cdots \stackrel{e_{n}}{\longrightarrow}\left(\ell_{n+1}, v_{n+1}\right) \cdots$ in $\operatorname{Runs}(G)$ is winning if there is some $k \geq 0$ s.t. $\left(\ell_{k}, v_{k}\right) \in K$ (resp. for all $k \geq 0$ s.t. $\left.\left(\ell_{k}, v_{k}\right) \in K\right)$. The set of winning runs in $G$ from $(\ell, v)$ is denoted WinRuns $((\ell, v), G)$.

For reachability games we assume w.l.o.g. that the goal is a particular location Goal. For safety games the goal is a set a locations to avoid.

The formal definition of the control problems is based on the definitions of strategies and outcomes. A strategy [16] is a function that during the course of the game constantly gives information as to what the controller should do in order to win the game. In a given situation, the strategy could suggest the controller to either i) "do a particular controllable action" or ii) "do nothing at this point in time, just wait" which will be denoted by the special symbol $\lambda$.

Definition 3 (Strategy). Let $G=\left(L, \ell_{0}\right.$, Act, $X, E$, Inv) be a TGA. A strategy $f$ over $G$ is a partial function from $\operatorname{Runs}(G)$ to $\operatorname{Act}_{c} \cup\{\lambda\}$ s.t. for every finite run $\rho$, if $f(\rho) \in \operatorname{Act}_{c}$ then last $(\rho) \stackrel{f(\rho)}{\longrightarrow} S_{G}\left(\ell^{\prime}, v^{\prime}\right)$ for some $\left(\ell^{\prime}, v^{\prime}\right)$.

We denote $\operatorname{Strat}(G)$ the set of strategies over $G$. A strategy $f$ is state-based whenever $\forall \rho, \rho^{\prime} \in \operatorname{Runs}(G)$, last $(\rho)=\operatorname{last}\left(\rho^{\prime}\right)$ implies that $f(\rho)=f\left(\rho^{\prime}\right)$. Statebased strategies are also called memoryless strategies in game theory $[9,19]$.

The restricted behavior of a TGA $G$ controlled with some strategy $f$ is defined by the notion of outcome [9].

Definition 4 (Outcome). Let $G=\left(L, \ell_{0}\right.$, Act, $\left.X, E, \operatorname{lnv}\right)$ be a TGA and $f$ a strategy over $G$. The outcome Outcome $(q, f)$ of $f$ from $q$ in $S_{G}$ is the subset of $\operatorname{Runs}(q, G)$ defined inductively by:

$-q \in$ Outcome $(q, f)$,

- if $\rho \in \operatorname{Outcome}(q, f)$ then $\rho^{\prime}=\rho \stackrel{e}{\longrightarrow} q^{\prime} \in \operatorname{Outcome}(q, f)$ if $\rho^{\prime} \in \operatorname{Runs}(q, G)$ and one of the following three conditions hold:

1. $e \in$ Act $_{u}$,

2. $e \in \operatorname{Act}_{c}$ and $e=f(\rho)$,

3. $e \in \mathbb{R}_{\geq 0}$ and $\forall 0 \leq e^{\prime}<e, \exists q^{\prime \prime} \in Q$ s.t. last $(\rho) \stackrel{e^{\prime}}{\longrightarrow} q^{\prime \prime} \wedge f\left(\rho \stackrel{e^{\prime}}{\longrightarrow} q^{\prime \prime}\right)=\lambda$. 
- for an infinite run $\rho, \rho \in \operatorname{Outcome}(q, f)$ if all the finite prefixes of $\rho$ are in Outcome $(q, f)$.

We assume that uncontrollable actions can only spoil the game and the controller has to do some controllable action to win $[6,16,11]$. In other words, an uncontrollable action cannot be forced to happen in $G$. Thus, a run may end in a state where only uncontrollable actions can be taken. Moreover we focus on reachability games and assume $K=\{$ Goal $\} \times \mathbb{R}_{\geq 0}^{X}$. A maximal run $\rho$ is either an infinite run (supposing no infinite sequence of delay transitions of duration 0 ) or a finite run $\rho$ that satisfies either i) $\operatorname{last}(\rho) \in K$ or ii) if $\rho \stackrel{a}{\longrightarrow}$ then $a \in \operatorname{Act}_{u}$ (i.e. the only possible next discrete actions from $\operatorname{last}(\rho)$, if any, are uncontrollable actions).

A strategy $f$ is winning from $q$ if all maximal runs in Outcome $(q, f)$ are in WinRuns $(q, G)$. A state $q$ in a TGA $G$ is winning if there exists a winning strategy $f$ from $q$ in $G$. We denote by $\mathcal{W}(G)$ the set of winning states in $G$ and WinStrat $(q, G)$ the set of winning strategies from $q$ over $G$.

\subsection{Backwards Algorithms for Solving Timed Games}

Let $G=\left(L, \ell_{0}\right.$, Act, $X, E$, Inv) be a TGA. For reachability games, the computation of the winning states is based on the definition of a controllable predecessor operator $[9,16]$. The controllable and uncontrollable discrete predecessors of $X$ are defined by cPred $(X)=\bigcup_{c \in \operatorname{Act}_{c}} \operatorname{Pred}_{c}(X)$ and uPred$(X)=\bigcup_{u \in \operatorname{Act}_{u}} \operatorname{Pred}_{u}(X)$. A notion of safe timed predecessors of a set $X$ w.r.t. a set $Y$ is also needed. Intuitively a state $q$ is in $\operatorname{Pred}_{t}(X, Y)$ if from $q$ we can reach $q^{\prime} \in X$ by time elapsing and along the path from $q$ to $q^{\prime}$ we avoid $Y$. Formally this is defined by:

$$
\operatorname{Pred}_{t}(X, Y)=\left\{q \in Q \mid \exists \delta \in \mathbb{R}_{\geq 0} \text { s.t. } q \stackrel{\delta}{\rightarrow} q^{\prime}, q^{\prime} \in X \text { and } \operatorname{Post}_{[0, \delta]}(q) \subseteq \bar{Y}\right\}
$$

where $\operatorname{Post}_{[0, \delta]}(q)=\left\{q^{\prime} \in Q \mid \exists t \in[0, \delta]\right.$ s.t. $\left.q \stackrel{t}{\longrightarrow} q^{\prime}\right\}$ and $\bar{Y}=Q \backslash Y$. The controllable predecessors operator $\pi$ is defined as follows ${ }^{4}$ :

$$
\pi(X)=\operatorname{Pred}_{t}(X \cup \mathrm{cPred}(X), \mathrm{uPred}(\bar{X}))
$$

Let $(G, K)$ be a reachability game, if $S$ is a finite union of symbolic states, then $\pi(S)$ is again a finite union of symbolic states. Moreover the iterative process given by $W^{0}=K$ and $W^{n+1}=\pi\left(W^{n}\right)$ will converge after finitely many steps for TGA [16] and the least fixed point obtained is $W^{*}$. It is also proved in [16] that $W^{*}=\mathcal{W}(G)$. Note also that $W^{*}$ is the maximal set of winning states of $G$ i.e. a state is winning iff it is in $W^{*}$. Thus there is a winning strategy in $G$ iff $\left(\ell_{0}, \overrightarrow{0}\right) \in W^{*}$. Altogether this gives a symbolic algorithm for solving reachability games. Extracting strategies can be done using the winning set of states $W^{*}$. For safety games $(G, K)$, it suffices to swap the roles of the players leading to a game $\bar{G}$ and solve a reachability game $(\bar{G}, \bar{K})$. If the winning set of states for $(\bar{G}, \bar{K})$ is $W$ then the winning set of states of $(G, K)$ is $\bar{W}$.

\footnotetext{
${ }^{4}$ Note that $\pi$ is defined here such that uncontrollable actions cannot be used to win.
} 


\section{On-the-fly Algorithm for Untimed Games}

For finite-state systems, on-the-fly model-checking algorithms has been an active and successful research area since the end of the 80's, with the algorithm proposed by Liu \& Smolka [15] being particularly elegant (and optimal). We present here our instantiation of this algorithm to untimed reachability games.

We consider untimed games as a restricted class of timed games with only finitely many states $Q$ and with only discrete actions, i.e. the set of labels is Act. Hence (memoryless) strategies simplifies to a choice of controllable action given the current state, i.e. $f: Q \rightarrow$ Act $_{c}$. For (untimed) reachability games we assume a designated set Goal of goal-states and the purpose of the analysis is to decide the existence of a strategy $f$ where all runs contains at least one state from Goal.

Now, our instantiation OTFUR of the local algorithm by Liu \& Smolka to untimed reachability games is given in Fig. 3. This algorithm is based on a waiting-list, Waiting $\subseteq E$ of edges waiting to be explored together with a passed-list Passed $\subseteq Q$ containing the states that have been encountered so far. Information about the current winning status of a state is given by a function Win : Passed $\rightarrow\{0,1\}$, where $\operatorname{Win}[q]$ is initialized to 0 and later potentially upgraded to 1 when the winning status of successors to $q$ change from 0 to 1 . To activate the reevaluation of the winning status of states, each state $q$ has an associated set of edges Depend $[q]$ depending on it: at any stage Depend $[q]$ contains all edges $\left(q^{\prime}, \alpha, q\right)$ that was encountered at a moment when $\operatorname{Win}[q]=$ 0 and where the winning status of the source state $q^{\prime}$ must be scheduled for reevaluation at the movement $\operatorname{Win}[q]=1$ becomes true. We refer to [15] for the formal proof of correctness of this algorithm summarized by the following theorem:

Theorem 1 ([15]). Upon termination of running the algorithm OTFUR on a given untimed game $G$ the following holds:

1. If $q \in$ Passed and Win $[q]=1$ then $q \in \mathcal{W}(G)$;

2. If Waiting $=\emptyset$ and Win $[q]=0$ then $q \notin \mathcal{W}(G)$.

In fact, the first property is an invariant of the while-statement holding after each iteration. Also, the algorithm is optimal in that it has linear time complexity in the size of the underlying untimed game: it is easy to see that each edge $e=\left(q, \alpha, q^{\prime}\right)$ will be added to Waiting at most twice, the first time $q$ is encountered (and added to Passed) and the second time when $\operatorname{Win}\left[q^{\prime}\right]$ changes winning status from 0 to $1^{5}$.

\footnotetext{
${ }^{5}$ To obtain an algorithm running in linear time in the size of $G$ (i.e. $|Q|+|E|$ ) it is important that the reevaluation of the winning status of a state $q$ does not directly involve (repeated and expensive) evaluation of the large boolean expression for $W i n^{*}$. In a practice, this may be avoided by adding a boolean $b_{q}$ and a counter $c_{q}$ recording the existence of a winning, controllable successor of $q$, and the number of winning, uncontrollable successor of $q$.
} 


\section{Initialization:}

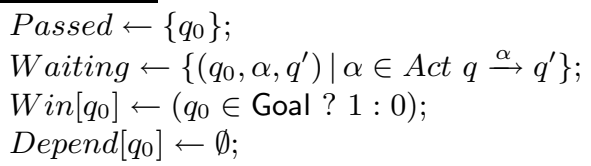

Main:

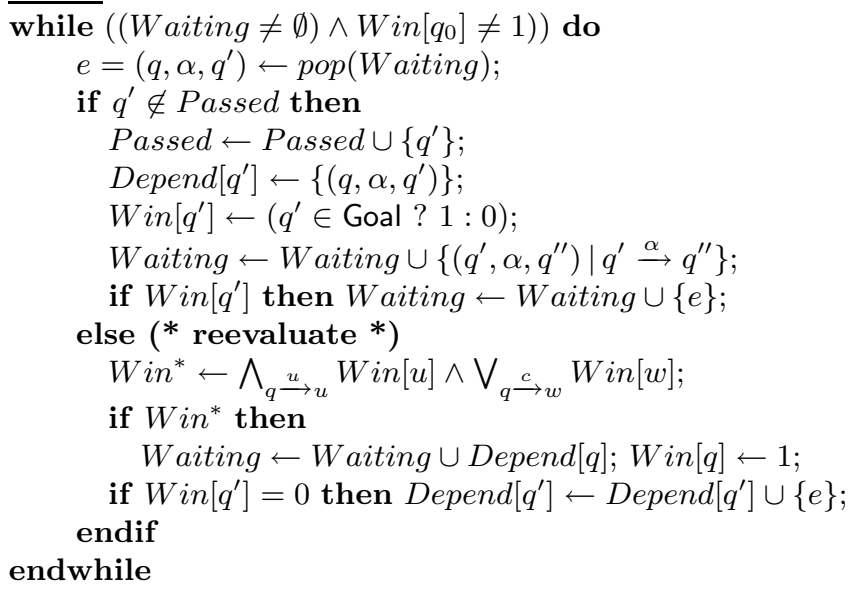

Fig. 3. OTFUR: On-The-Fly Algorithm for Untimed Reachability Games

\section{On-the-fly Algorithm for Timed Games}

Now let us turn our attention to the timed case and present our symbolic extension of the algorithm of Liu \& Smolka providing a zone-based forward and onthe-fly algorithm for solving timed reachability games. The algorithm, SOTFTR, is given in Fig. 4 and may be viewed as an interleaved combination of forward computation of the simulation graph of the timed game automaton together with back-propagation of information of winning states. As in the untimed case the algorithm is based on a waiting-list, Waiting, of edges in the simulation-graph to be explored, and a passed-list, Passed, containing all the symbolic states of the simulation-graph encountered so far by the algorithm.

The crucial point of our symbolic extension is that the winning status $W i n[q]$ of an individual state $q$ is replaced by a set $\operatorname{Win}[S] \subseteq S$ identifying the subset of the symbolic state $S$ which is currently known to be winning. The set Depend $[S]$ indicates the set of edges (or predecessors of $S$ ) which must be reevaluated (i.e. added to Waiting) when new information about $W i n[S]$ is obtained (i.e. when $\left.\operatorname{Win}[S] \subsetneq W_{i n}^{*}\right)$. Whenever an edge $e=\left(S, \alpha, S^{\prime}\right)$ is considered with $S^{\prime} \in$ Passed, the edge $e$ is added to the dependency set of $S^{\prime}$ in order that possible future information about additional winning states within $S^{\prime}$ may also be back-propagated to $S$. In Table 1, we illustrate the forward exploration and backwards propagation steps of the algorithm. 


\section{Initialization:}

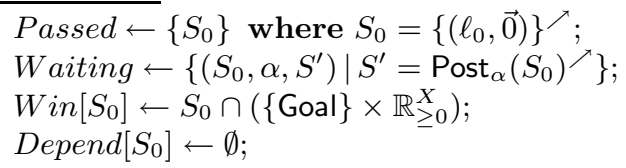

Main:

while $\left((W\right.$ aiting $\neq \emptyset) \wedge\left(s_{0} \notin W\right.$ in $\left.\left.\left[S_{0}\right]\right)\right)$ do $e=\left(S, \alpha, S^{\prime}\right) \leftarrow \operatorname{pop}($ Waiting $)$;

if $S^{\prime} \notin$ Passed then Passed $\leftarrow$ Passed $\cup\left\{S^{\prime}\right\} ;$ Depend $\left[S^{\prime}\right] \leftarrow\left\{\left(S, \alpha, S^{\prime}\right)\right\}$; Win $\left[S^{\prime}\right] \leftarrow S^{\prime} \cap\left(\{\right.$ Goal $\left.\} \times \mathbb{R}_{\geq 0}^{X}\right)$;

Waiting $\leftarrow$ Waiting $\cup\left\{\left(S^{\prime}, \alpha, S^{\prime \prime}\right) \mid S^{\prime \prime}=\operatorname{Post}_{\alpha}\left(S^{\prime}\right)^{\nearrow}\right\}$; if $W i n\left[S^{\prime}\right] \neq \emptyset$ then Waiting $\leftarrow$ Waiting $\cup\{e\}$; else $(* \text { reevaluate } *)^{a}$

Win $^{*} \leftarrow \operatorname{Pred}_{t}\left(\right.$ Win $[S] \cup \bigcup_{S \stackrel{c}{\rightarrow} T} \operatorname{Pred}_{c}($ Win $[T])$,

if $\left(\right.$ Win $\left.[S] \subsetneq W i n^{*}\right)$ then $\left.\bigcup_{S} \stackrel{u}{\rightarrow} T \operatorname{Pred}_{u}(T \backslash W i n[T])\right) \cap S$

Waiting $\leftarrow$ Waiting $\cup$ Depend $[S] ;$ Win $[S] \leftarrow W i n^{*}$; Depend $\left[S^{\prime}\right] \leftarrow$ Depend $\left[S^{\prime}\right] \cup\{e\}$; endif

endwhile

${ }^{a}$ When $T \notin$ Passed,Win $[T]=\emptyset$

Fig. 4. SOTFTR: Symbolic On-The-Fly Algorithm for Timed Reachability Games

The correctness of the symbolic on-the-fly algorithm SOTFTR is given by the following lemma and theorem, the rigorous proofs of which can be found in the appendix. 


\begin{tabular}{|c|c|c|c|c|c|c|}
\hline \multicolumn{3}{|c|}{ Steps } & \multirow[t]{2}{*}{ Waiting } & \multirow[t]{2}{*}{ Passed } & \multirow[t]{2}{*}{ Depend } & \multirow[t]{2}{*}{ Win } \\
\hline$\#$ & $S$ & $S^{\prime}$ & & & & \\
\hline 0 & - & - & $\left(S_{0}, u_{1}, S_{1}\right),\left(S_{0}, u_{2}, S_{2}\right),\left(\mathbf{S}_{\mathbf{0}}, \mathbf{c}_{1}, \mathbf{S}_{\mathbf{3}}\right)$ & $\overline{S_{0}}$ & - & $\left(S_{0}, \emptyset\right)$ \\
\hline 1 & $S_{0}$ & $S_{3}$ & $\begin{array}{l}\left(S_{0}, u_{1}, S_{1}\right),\left(S_{0}, u_{2}, S_{2}\right) \\
+\left(\mathbf{S}_{\mathbf{3}}, \mathbf{c}_{\mathbf{1}}, \mathbf{S}_{\mathbf{4}}\right),\left(S_{3}, u_{3}, S_{2}\right)\end{array}$ & $S_{3}$ & $S_{3} \mapsto\left(S_{0}, c_{1}, S_{3}\right)$ & $\left(S_{3}, \emptyset\right)$ \\
\hline 2 & $S_{3}$ & $S_{4}$ & $\begin{array}{l}\left(S_{0}, u_{1}, S_{1}\right),\left(S_{0}, u_{2}, S_{2}\right),\left(S_{3}, u_{3}, S_{2}\right) \\
+\left(\mathbf{S}_{\mathbf{3}}, \mathbf{c}_{\mathbf{2}}, \mathbf{S}_{\mathbf{4}}\right)\end{array}$ & $S_{4}$ & $S_{4} \mapsto\left(S_{3}, c_{2}, S_{4}\right)$ & $\left(S_{4}, S_{4}\right)$ \\
\hline 3 & $S_{3}$ & $S_{4}$ & $\begin{array}{l}\left(S_{0}, u_{1}, S_{1}\right),\left(S_{0}, u_{2}, S_{2}\right),\left(S_{3}, u_{3}, S_{2}\right) \\
+\left(\mathbf{S}_{\mathbf{0}}, \mathbf{c}_{\mathbf{1}}, \mathbf{S}_{\mathbf{3}}\right)\end{array}$ & - & - & $\left(S_{3}, x \geq 1\right)$ \\
\hline 4 & $S_{0}$ & $S_{3}$ & $\left(S_{0}, u_{1}, S_{1}\right),\left(S_{0}, u_{2}, S_{2}\right),\left(\mathbf{S}_{\mathbf{3}}, \mathbf{u}_{\mathbf{3}}, \mathbf{S}_{\mathbf{2}}\right)$ & $S_{4}$ & $S_{3} \mapsto\left(S_{0}, c_{1}, S_{3}\right)$ & $\left(S_{0}, x=1\right)$ \\
\hline 5 & $S_{3}$ & $S_{2}$ & \begin{tabular}{|l} 
\\
$\quad\left(S_{0}, u_{1}, S_{1}\right),\left(S_{0}, u_{2}, S_{2}\right)$ \\
$+\left(\mathbf{S}_{\mathbf{2}}, \mathbf{c}_{\mathbf{3}}, \mathbf{S}_{\mathbf{5}}\right)$ \\
\end{tabular} & $S_{2}$ & $S_{2} \mapsto\left(S_{3}, u_{3}, S_{2}\right)$ & $\left(S_{2}, \emptyset\right)$ \\
\hline 6 & $S_{2}$ & $S_{5}$ & $\begin{array}{l}\left(S_{0}, u_{1}, S_{1}\right),\left(S_{0}, u_{2}, S_{2}\right) \\
+\left(\mathbf{S}_{\mathbf{5}}, \mathbf{c}_{\mathbf{4}}, \mathbf{S}_{\mathbf{3}}\right) \\
\end{array}$ & $S_{5}$ & $S_{5} \mapsto\left(S_{2}, c_{3}, S_{2}\right)$ & $\left(S_{5}, \emptyset\right)$ \\
\hline 7 & $S_{5}$ & $S_{3}$ & $\begin{array}{l}\left(S_{0}, u_{1}, S_{1}\right),\left(S_{0}, u_{2}, S_{2}\right) \\
+\left(\mathbf{S}_{\mathbf{2}}, \mathbf{c}_{\mathbf{3}}, \mathbf{S}_{\mathbf{5}}\right)\end{array}$ & - & $\begin{aligned} S_{3} \mapsto \begin{array}{l}\left(S_{2}, c_{3}, S_{2}\right) \\
\left(S_{5}, c_{4}, S_{3}\right)\end{array} \\
\end{aligned}$ & $\left(S_{5}, x \leq 1\right)$ \\
\hline 8 & $S_{2}$ & $S_{5}$ & $\begin{array}{l}\left(S_{0}, u_{1}, S_{1}\right),\left(S_{0}, u_{2}, S_{2}\right) \\
+\left(\mathbf{S}_{\mathbf{3}}, \mathbf{u}_{\mathbf{3}}, \mathbf{S}_{\mathbf{2}}\right)\end{array}$ & - & $S_{5} \mapsto\left(S_{2}, c_{3}, S_{2}\right)$ & $\left(S_{2}, x \leq 1\right)$ \\
\hline 9 & $S_{3}$ & $S_{2}$ & $\begin{array}{l}\left(S_{0}, u_{1}, S_{1}\right),\left(\mathbf{S}_{\mathbf{0}}, \mathbf{u}_{\mathbf{2}}, \mathbf{S}_{\mathbf{2}}\right) \\
+\left(S_{0}, c_{1}, S_{3}\right),\left(S_{5}, c_{4}, S_{3}\right) \\
\end{array}$ & - & - & $\left(S_{3}, S_{3}\right)$ \\
\hline 10 & $S_{0}$ & $S_{2}$ & $\left(S_{0}, u_{1}, S_{1}\right),\left(S_{0}, c_{1}, S_{3}\right),\left(\mathbf{S}_{\mathbf{5}}, \mathbf{c}_{\mathbf{4}}, \mathbf{S}_{\mathbf{3}}\right)$ & - & $\begin{aligned} S_{2} \mapsto \begin{array}{l}\left(S_{3}, u_{3}, S_{2}\right) \\
\left(S_{0}, u_{2}, S_{2}\right)\end{array} \\
\end{aligned}$ & $\left(S_{0}, x \leq 1\right)$ \\
\hline 11 & $S_{5}$ & $S_{3}$ & $\left(S_{0}, u_{1}, S_{1}\right),\left(\mathbf{S}_{\mathbf{0}}, \mathbf{c}_{\mathbf{1}}, \mathbf{S}_{\mathbf{3}}\right)$ & - & - & - \\
\hline 12 & $S_{0}$ & $S_{3}$ & $\left(\mathbf{S}_{\mathbf{0}}, \mathbf{u}_{1}, \mathbf{S}_{1}\right)$ & - & - & - \\
\hline 13 & $S_{0}$ & $S_{1}$ & $\emptyset$ & $S_{1}$ & $S_{1} \mapsto\left(S_{0}, u_{1}, S_{1}\right)$ & $\left(S_{1}, \emptyset\right)$ \\
\hline
\end{tabular}

At step $n,\left(\mathbf{S}, \alpha, \mathbf{S}^{\prime}\right)$ is the transition popped at step $n+1$;

At step $n,+\left(S, \alpha, S^{\prime}\right)$ the transition added to Waiting at step $n$;

Symbolic States: $S_{0}=\left(\ell_{1}, x \geq 0\right), S_{1}=\left(\ell_{5}, x>1\right), S_{2}=\left(\ell_{3}, x \geq 0\right), S_{3}=\left(\ell_{2}, x \geq 0\right)$,

$S_{4}=($ Goal, $x \geq 2), S_{5}=\left(\ell_{4}, \bar{x} \geq 0\right)$

Table 1. Running SOTFTG

Lemma 1. The while-loop of algorithm SOTFTR has the following invariance properties when running on a timed game automaton $G$ :

1. For any $S \in$ Passed if $S \stackrel{\alpha}{\longrightarrow} S^{\prime}$ then either $\left(S, \alpha, S^{\prime}\right) \in$ Waiting or $S^{\prime} \in$ Passed and $\left(S, \alpha, S^{\prime}\right) \in$ Depend $\left[S^{\prime}\right]$

2. If $q \in W i n[S]$ for some $S \in$ Passed then $q \in \mathcal{W}(G)$

3. If $q \in S \backslash W i n[S]$ for some $S \in$ Passed then either

$-e \in W$ aiting for some $e=\left(S, \alpha, S^{\prime}\right)$ with $S^{\prime} \in$ Passed, or

- $q \notin \operatorname{Pred}_{t}\left[\right.$ Win $\left.[S] \cup \bigcup_{S \stackrel{c}{\rightarrow} T} \operatorname{Pred}_{c}(\operatorname{Win}[T]), \bigcup_{S} \stackrel{u}{\rightarrow}_{T} \operatorname{Pred}_{u}(T \backslash W i n[T])\right]$.

Theorem 2. Upon termination of running the algorithm SOTFTR on a given timed game automaton $G$ the following holds:

1. If $q \in W i n[S]$ for some $S \in$ Passed then $q \in \mathcal{W}(G)$;

2. If Waiting $=\emptyset, q \in S \backslash W i n[S]$ for some $S \in$ Passed then $q \notin \mathcal{W}(G)$.

Termination of the algorithm SOTFTR is guaranteed by the finiteness of symbolic states ${ }^{6}$ and the fact that each edge $(S, \alpha, T)$ will be present in the Waiting-list at most $1+|T|$ times, where $|T|$ is the number of regions of $T$ :

${ }^{6}$ Strictly speaking, this requires that we either transforms the given TGA into an equivalent one in which all location-invariants insist on an upper bound on all clocks or, alternatively, that we apply standard extrapolation w.r.t. maximal constant occurring in the TGA (which is correct up to time-abstracted bisimulation). 
$(S, \alpha, T)$ will be in Waiting the first time that $S$ is encountered and subsequently each time the value of $W i n[T]$ increases. Now, any given region may be contained in several symbolic states of the simulation graph (due to overlap). Thus the SOTFTR algorithm is not linear in the region-graph and hence not theoretically optimal, as an algorithm with linear worst-case time-complexity could be obtained by applying the untimed algorithm directly to the regiongraph. However, this is only a theoretical result and, as we shall see, the use of zones yields very encouraging performance results in practice, as is the case for reachability analysis of timed automata.

\section{Implementation, Optimizations and Extensions}

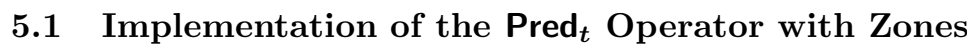

In order to be efficient, the algorithm SOTFTR manipulates zones. However, while a forward step always gives a single zone as a result, the Pred Poperator does not. So, given a symbolic state $S, \operatorname{Win}[S]$ is, in general, an union of zones (and so is $S \backslash W i n[S]$ ). As a consequence, we now give two results, which allow us to handle unions of zones (Theorem 3) and to define the computation of Pred $t$ in terms of basic operations on zones (Theorem 4).

Theorem 3. The following distribution law holds:

$$
\operatorname{Pred}_{t}\left(\bigcup_{i} G_{i}, \bigcup_{j} B_{j}\right)=\bigcup_{i} \bigcap_{j} \operatorname{Pred}_{t}\left(G_{i}, B\right)
$$

Theorem 4. If $B$ is a convex set, then the $\operatorname{Pred}_{t}$ operator defined in equation (1) can be expressed as:

$$
\operatorname{Pred}_{t}(G, B)=\left(G^{\swarrow} \backslash B^{\swarrow}\right) \cup\left(\left(G \cap B^{\swarrow}\right) \backslash B\right)^{\swarrow}
$$

\subsection{Optimizations}

Zone Inclusion. When we explore forward the automaton, we check if any newly generated symbolic state $S^{\prime}$ belongs to the passed list: $S^{\prime} \in$ Passed. As an optimization we may instead use the classical inclusion check: $\exists S^{\prime \prime} \in$ Passed s.t. $S^{\prime} \subseteq S^{\prime \prime}$, in which case, $S^{\prime}$ is discarded and we update the dependency graph as well. Indeed, new information learned from the successors of $S^{\prime \prime}$ can be new information on $S^{\prime}$ but not necessarily. This introduces an overhead in the sense that we may back-propagate information for nothing.

On the other hand, back-propagating only the relevant information would be unnecessarily complex and would void most of the memory gain introduced by the use of inclusion. In practice, the reduction of the number of forward steps

obtained by the inclusion check pays off for large systems and is a little overhead otherwise, as shown in our experiments. 
Losing states. In the case of reachability games we can sometimes decide at an early stage that a state $q$ is losing (i.e. $q \notin \mathcal{W}\left(S_{G}\right)$ ), either because it is given as a part of the model in the same way as goal states, or because it is deadlock state, which is not in the set of goal states.

The detection of such losing states has a two-fold benefit. First, we can stop the forward exploration on these states, since we know that we have lost (in the case of a user-defined non-deadlock losing state). Second, we can back-propagate these losing states in the same way as we do for winning states and stop the algorithm if we have the initial state $s_{0} \in \operatorname{Lose}\left[S_{0}\right]$, where $\operatorname{Lose}[S]$ is the subset of the symbolic state $S$ currently known to be losing. In some cases, this can bring a big benefit, illustrated by Fig. 1 , if the guard $x<1$ is changed to true in the edge from $\ell_{1}$ to $\ell_{5}$.

Pruning. In the basic algorithm early termination takes place when the initial state is known to be winning (i.e. $\left.s_{0} \in \operatorname{Win}\left[S_{0}\right]\right)$. However, we may extend this principle to other parts of the algorithm. In particular, we can add the condition that whenever an edge $e=\left(S, \alpha, S^{\prime}\right)$ is selected and it turns out that $\operatorname{Win}[S]=S$ then we may safely skip the rest of the while loop as we know that no further knowledge on the winning states of $S$ can be gained. In doing so, we prune unnecessary continued forward exploration and/or expensive reevaluation. When we back-propagate losing states as described previously, the condition naturally extends to $\operatorname{Win}[S] \cup \operatorname{Lose}[S]=S$.

\subsection{Time Optimal Strategy Synthesis}

Time-optimality for reachability games consists in computing the best (optimal) time the controller can guarantee to reach the Goal location: if $t^{*}$ is the optimaltime, the controller has a strategy that guarantees to reach location Goal within $t^{*}$ time units whatever the opponent is doing, and moreover, the controller has no strategy to guarantee this for any $t<t^{*}$.

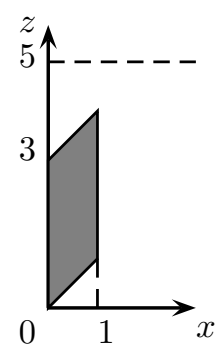

Fig. 5. Winning subset of the initial zone of the TGA of Fig. 1 with clock $z$ added.
First consider the following problem: decide whether the controller has a strategy to reach location Goal within $B$ time units. To solve this problem, we just add a fresh clock $z$ to the TGA $G$ and the invariant $\operatorname{lnv}(\ell) \equiv z \leq B$ for all locations $\ell$ with $z$ being unconstrained in the initial state. Then we compute the set of winning states of this game and check that $\left(\ell_{0}, \overrightarrow{0}, z=0\right)$ is actually a winning state. If not, try with some $B^{\prime}>B$. Otherwise we know that the controller can guarantee to reach Goal within $B$ time units ... but in addition we have the optimal-time to reach Goal ${ }^{7}$. Indeed, when computing the winning set of states $W^{*}$ on the TGA $G$ augmented with the $z$ clock (being initially unconstrained), we have the maximal set of winning states. This means

\footnotetext{
7 To get an optimum, the condition of the while-loop must be Waiting $\neq \emptyset$ alone in the algorithm, disabling early termination.
} 
that we obtain some $\left(\ell_{0}, Z_{0}\right) \in W^{*}$ and $\left(\ell_{0}, \overrightarrow{0}, z=0\right) \in\left(\ell_{0}, Z_{0}\right)$. But $Z_{0} \cap\left\{\left(\ell_{0}, \overrightarrow{0}\right)\right\}$ gives us for free the optimal-time to reach Goal. Assume $I=Z_{0} \cap\left\{\left(\ell_{0}, \overrightarrow{0}\right)\right\}$, then $0 \in I$ and the upper bound of $I$ is less than $B$. This means that starting in $\left(\ell_{0}, \overrightarrow{0}\right)$ with $z \in I$ the controller can guarantee to reach Goal within $B$ time units. And as $W^{*}$ is the maximal set of winning states, starting with $z \notin I$ cannot guarantee this any more. Assume $I=[0, b]$. The optimal-time is then $t^{*}=B-b$. If it turns out that $I$ is right open $[0, b[$, we even know more: that this optimal time $t^{*}$ cannot be achieved by any strategy, but we can reach Goal in a time arbitrarily close to $t^{*}$. On the example of Fig. 1 , if we choose $B=5$ we obtain a closed interval $I=[0,3]$ giving the optimal time $t^{*}=2$ to reach Goal (Fig. 5). Moreover we know that there is a strategy that guarantees this optimal.

\section{Experiments}

Several versions of the described timed game reachability algorithm have been implemented: with or without inclusion checking between zones, with or without back-propagation of the losing states, and with or without pruning. To benchmark the implementations we used the Production Cell [14,17] case study (Fig. 6). Unprocessed plates arrive on a feeding belt, are taken by a robot to a press, are processed, and are taken away to a departure belt. The robot has two $\operatorname{arms}(\mathrm{A}$ and $\mathrm{B})$ to take and release the plates and its actions are controllable, except for the time needed to rotate. The arrival of the plates and the press are uncontrollable.

We run experiments on a dualXeon $2.8 \mathrm{GHz}$ equipped with $3 \mathrm{~GB}$ of RAM running Linux 2.4.21. Table 2 shows the obtained results. The tests are done with varying number of plates from 2 to 7 , and with controllable (win) and uncontrollable (lose) configurations. The models contain from 4 to 10 clocks.

The inclusion checking of zones is shown to be an important optimization. Furthermore, activating pruning, which really exploits

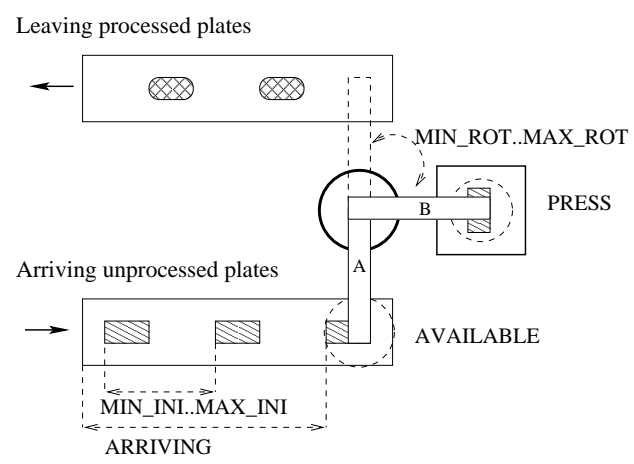

Fig. 6. The production cell that the algorithm is on-the-fly, is useful in practice: the algorithm really terminates earlier. The results for time optimal reachability confirm that the algorithm is exploring the whole state-space and is comparable to exploring without pruning. We stress in the tables the best result obtained for every configuration: it turns out that propagating back the losing states has a significant overhead that pays off for large systems, i.e. it is clearly better from 6 plates.

The state-space grows exponentially with respect to the number of plates but the algorithm keeps up linearly with it, which is shown on Fig. 7 that depicts 


\begin{tabular}{|c|c|c|c|c|c|c|c|c|c|c|c|}
\hline \multicolumn{2}{|c|}{ Plates } & \multicolumn{2}{|c|}{ Basic } & \multicolumn{2}{|c|}{ Basic +inc } & \multicolumn{2}{|c|}{$\begin{array}{c}\text { Basic +inc } \\
\text { +pruning }\end{array}$} & \multicolumn{2}{|c|}{$\begin{array}{c}\text { Basic +lose +inc } \\
\text { +pruning }\end{array}$} & \multicolumn{2}{|c|}{$\begin{array}{c}\text { Basic +lose +inc } \\
+ \text { topt }\end{array}$} \\
\hline \multirow{3}{*}{2} & & time & mem & time & mem & time & mem & $\overline{\text { time }}$ & mem & time & mem \\
\hline & win & $0.0 \mathrm{~s}$ & $1 \mathrm{M}$ & $0.0 \mathrm{~s}$ & $1 \mathrm{M}$ & $0.0 \mathrm{~s}$ & $1 \mathrm{M}$ & $0.0 \mathrm{~s}$ & $1 \mathrm{M}$ & $0.04 \mathrm{~s}$ & $1 \mathrm{M}$ \\
\hline & lose & $0.0 \mathrm{~s}$ & $1 \mathrm{M}$ & $0.0 \mathrm{~s}$ & $1 \mathrm{M}$ & $0.0 \mathrm{~s}$ & $1 \mathrm{M}$ & $0.0 \mathrm{~s}$ & $1 \mathrm{M}$ & $\mathrm{n} / \mathrm{a}$ & $\mathrm{n} / \mathrm{a}$ \\
\hline \multirow{2}{*}{3} & win & $0.5 \mathrm{~s}$ & $19 \mathrm{M}$ & $0.0 \mathrm{~s}$ & $1 \mathrm{M}$ & $0.0 \mathrm{~s}$ & $1 \mathrm{M}$ & $0.1 \mathrm{~s}$ & $1 \mathrm{M}$ & $0.27 \mathrm{~s}$ & $4 \mathrm{M}$ \\
\hline & lose & $1.1 \mathrm{~s}$ & $45 \mathrm{M}$ & $0.1 \mathrm{~s}$ & $1 \mathrm{M}$ & $0.0 \mathrm{~s}$ & $1 \mathrm{M}$ & $0.2 \mathrm{~s}$ & $3 \mathrm{M}$ & $\mathrm{n} / \mathrm{a}$ & $\mathrm{n} / \mathrm{a}$ \\
\hline \multirow{2}{*}{4} & win & $33.9 \mathrm{~s}$ & $1395 \mathrm{M}$ & $0.2 \mathrm{~s}$ & $8 \mathrm{M}$ & $0.1 \mathrm{~s}$ & $6 \mathrm{M}$ & $0.4 \mathrm{~s}$ & $5 \mathrm{M}$ & $1.88 \mathrm{~s}$ & $13 \mathrm{M}$ \\
\hline & lose & - & - & $0.5 \mathrm{~s}$ & $11 \mathrm{M}$ & $0.4 \mathrm{~s}$ & $10 \mathrm{M}$ & $0.9 \mathrm{~s}$ & $\overline{9 M}$ & $\mathrm{n} / \mathrm{a}$ & $\mathrm{n} / \mathrm{a}$ \\
\hline \multirow[t]{2}{*}{5} & win & - & - & $3.0 \mathrm{~s}$ & $31 \mathrm{M}$ & $1.5 \mathrm{~s}$ & $\overline{22 \mathrm{M}}$ & $2.0 \mathrm{~s}$ & $16 \mathrm{M}$ & $13.35 \mathrm{~s}$ & $59 \mathrm{M}$ \\
\hline & lose & - & - & $11.1 \mathrm{~s}$ & $61 \mathrm{M}$ & $5.9 \mathrm{~s}$ & $46 \mathrm{M}$ & $7.0 \mathrm{~s}$ & $41 \mathrm{M}$ & $\mathrm{n} / \mathrm{a}$ & $\mathrm{n} / \mathrm{a}$ \\
\hline \multirow[t]{2}{*}{6} & win & - & - & $89.1 \mathrm{~s}$ & $179 \mathrm{M}$ & $38.9 \mathrm{~s}$ & $121 \mathrm{M}$ & $12.0 \mathrm{~s}$ & $63 \mathrm{M}$ & $220.3 \mathrm{~s}$ & $369 \mathrm{M}$ \\
\hline & lose & - & - & $699 \mathrm{~s}$ & $480 \mathrm{M}$ & $317 \mathrm{~s}$ & $346 \mathrm{M}$ & 135.1s & 273M & $\mathrm{n} / \mathrm{a}$ & $\mathrm{n} / \mathrm{a}$ \\
\hline \multirow{2}{*}{7} & win & - & - & $3256 \mathrm{~s}$ & $1183 \mathrm{M}$ & $1181 \mathrm{~s}$ & $786 \mathrm{M}$ & $124 \mathrm{~s}$ & $319 \mathrm{M}$ & $6188 \mathrm{~s}$ & $2457 \mathrm{M}$ \\
\hline & lose & - & - & - & - & $16791 \mathrm{~s}$ & $2981 \mathrm{M}$ & $4075 \mathrm{~s}$ & $2090 M$ & $\mathrm{n} / \mathrm{a}$ & $\mathrm{n} / \mathrm{a}$ \\
\hline
\end{tabular}

Table 2. Results for the different implementations: basic algorithm, then with inclusion checking (inc), pruning (pruning), back propagation of losing states (lose) and time optimal strategy generation (topt, only for "win", and pruning has little effect). Time (user process) is given in seconds (s) rounded to 0.1s and memory in megabytes (M). '-' denotes a failed run (not enough memory). Results in bold font are the best ones.

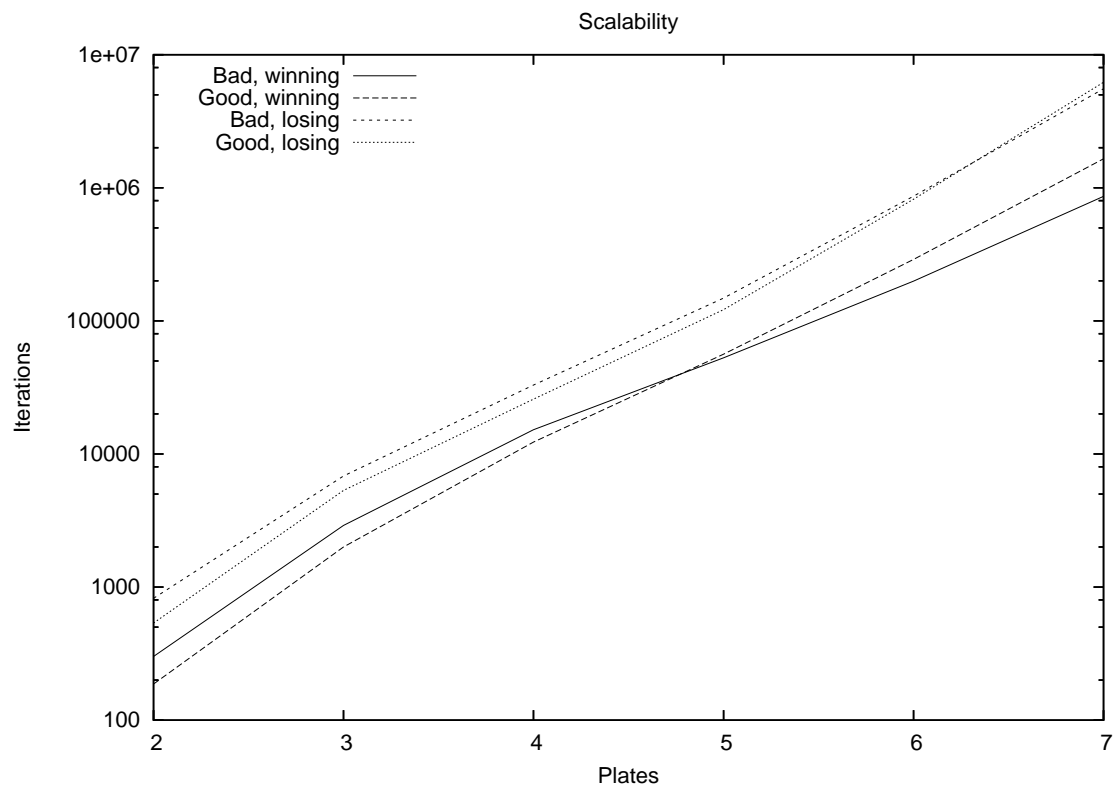

Fig. 7. Scalability of the algorithm. The scale is logarithmic. 
pre + post $^{8}$. These results show that the algorithm based on zones behaves well despite the fact that zones are (in theory) worse than regions.

\section{Conclusion and Future Work}

In this paper we have introduced what we believe is the first completely on-thefly algorithm for solving timed games. For its efficient implementation we have used zones as the main datastructure, and we have applied decisive optimizations to take full advantage of the on-the-fly nature of our algorithm and its zone representation. Experiments have shown that an implementation based on zones is feasible and with encouraging performances w.r.t. the complexity of the problem. Finally, we have exhibited how to obtain the time optimal strategies with minor additions to our algorithm (essentially book-keeping).

We are working on an improved version of the implementation to distribute it and use the UPPAAL GUI augmented with (un)controllable transitions. We are investigating more aggressive abstractions for the underlying simulation graph computed by our algorithm and efficient guiding of the search, in particular for optimal strategies. Our algorithm is well suited for distributed implementation by its use of unordered waiting-list and there are plans to pursue this directions as has been done for UPPAAL [7]. We are also investigating how to extract strategies and represent them compactly with CDDs (Clock Decision Diagrams).

Acknowledgments. The authors want to thank Patricia Bouyer and Gerd Behrmann for inspiring discussions on the topic of timed games.

\section{References}

1. K. Altisen and S. Tripakis. Tools for controller synthesis of timed systems. In Proc. 2nd Work. on Real-Time Tools (RT-TOOLS'02), 2002. Proc. published as Technical Report 2002-025, Uppsala University, Sweden.

2. R. Alur and D. Dill. A theory of timed automata. Theoretical Computer Science, 126(2):183-235, 1994.

3. R. Alur, S. La Torre, and G. J. Pappas. Optimal Paths in Weighted Timed Automata. In Proc. of $4^{\text {th }}$ Work. Hybrid Systems: Computation and Control (HSCC'01), volume 2034 of LNCS, pages 49-62. Springer, 2001.

4. Henrik R. Andersen. Model Checking and Boolean Graphs. Theoretical Computer Science, 126(1):3-30, 1994.

5. E. Asarin and O. Maler. As Soon as Possible: Time Optimal Control for Timed Automata. In Proc. 2nd Work. Hybrid Systems: Computation \& Control (HSCC'99), volume 1569 of LNCS, pages 19-30. Springer, 1999.

6. E. Asarin, O. Maler, A. Pnueli, and J. Sifakis. Controller Synthesis for Timed Automata. In Proc. IFAC Symp. on System Structure $\mathscr{E}$ Control, pages 469-474. Elsevier Science, 1998.

7. Gerd Behrmann. Distributed reachability analysis in timed automata. Journal of Software Tools for Technology Transfer (STTT), 7(1):19-30, 2005.

${ }^{8}$ pre + post represents the number of iterations of the algorithm and is therefore an abstraction in both time and space of the implementation. 
8. M. Bozga, C. Daws, O. Maler, A. Olivero, S. Tripakis, and S. Yovine. Kronos: a Model-Checking Tool for Real-Time Systems. In Proc. $10^{\text {th }}$ Conf. on Computer Aided Verification (CAV'98), volume 1427 of $L N C S$, pages 546-550. Springer, 1998.

9. L. De Alfaro, T. A. Henzinger, and R. Majumdar. Symbolic Algorithms for InfiniteState Games. In Proc. 12 ${ }^{\text {th }}$ Conf. on Concurrency Theory (CONCUR'01), volume 2154 of $L N C S$, pages 536-550. Springer, 2001.

10. Gerard J. Holzmann. The SPIN Model Checker. Addison-Wesley, 2003.

11. S. La Torre, S. Mukhopadhyay, and A. Murano. Optimal-Reachability and Control for Acyclic Weighted Timed Automata. In Proc. $2^{\text {nd }}$ IFIP Conf. on Theoretical Computer Science (TCS 2002), volume 223, pages 485-497. Kluwer, 2002.

12. K. G. Larsen. Efficient Local Correctness Checking. In Proc. of Conf. of Computer Assisted Verification (CAV'92), volume 663 of $L N C S$, pages 30-43. Springer, 1992.

13. K. G. Larsen, P. Pettersson, and W. Yi. UpPAAL in a Nutshell. Journal of Software Tools for Technology Transfer (STTT), 1(1-2):134-152, 1997.

14. C. Lewerentz and T. Lindner. Production Cell: A Comparative Study in Formal Specification and Verification. In Methods, Languages 86 Tools for Construction of Correct Software, volume 1009 of LNCS, pages 388-416. Springer, 1995.

15. X. Liu and S. Smolka. Simple Linear-Time Algorithm for Minimal Fixed Points. In Proc. 26 $6^{\text {th }}$ Conf. on Automata, Languages and Programming (ICALP'98), volume 1443 of $L N C S$, pages 53-66. Springer, 1998.

16. O. Maler, A. Pnueli, and J. Sifakis. On the synthesis of discrete controllers for timed systems. In Proc. $12^{\text {th }}$ Symp. on Theoretical Aspects of Computer Science (STACS'95), volume 900, pages 229-242. Springer, 1995.

17. H. Melcher and K. Winkelmann. Controller Synthesis for the "Production Cell" Case Study. In Proc. of $2^{\text {nd }}$ Work. on Formal Methods in Software Practice, pages 24-36. ACM Press, 1998.

18. J. Rasmussen, K. G. Larsen, and K. Subramani. Resource-optimal scheduling using priced timed automata. In Proc. $10^{\text {th }}$ Conf. on Tools and Algorithms for the Construction and Analysis of Systems (TACAS'04), volume 2988 of LNCS, pages 220-235. Springer, 2004.

19. W. Thomas. On the Synthesis of Strategies in Infinite Games. In Proc. 12 $2^{\text {th }}$ Symp. on Theoretical Aspects of Computer Science (STACS'95), volume 900, pages 1-13. Springer, 1995. Invited talk.

20. S. Tripakis and K. Altisen. On-the-Fly Controller Synthesis for Discrete and Timed Systems. In Proc. of World Congress on Formal Methods (FM'99), volume 1708 of LNCS, pages 233-252. Springer, 1999. 


\section{A Proofs of Theorems}

Lemma 1. The while-loop of algorithm SOTFTR has the following invariance properties when running on a timed game automaton $G$ :

1. For any $S \in$ Passed if $S \stackrel{\alpha}{\rightarrow} S^{\prime}$ then either $\left(S, \alpha, S^{\prime}\right) \in$ Waiting or $S^{\prime} \in$ Passed and $\left(S, \alpha, S^{\prime}\right) \in$ Depend $\left[S^{\prime}\right]$.

2. If $q \in W$ in $[S]$ for some $S \in$ Passed then $q \in \mathcal{W}(G)$

3. If $q \in S \backslash W i n[S]$ for some $S \in$ Passed then either

$-e \in W$ aiting for some $e=\left(S, \alpha, S^{\prime}\right)$ with $S^{\prime} \in$ Passed,

or

- $q \notin \operatorname{Pred}_{t}\left[\operatorname{Win}[S] \cup \bigcup_{S \stackrel{c}{\rightarrow} T} \operatorname{Pred}_{c}\left(\operatorname{Win}_{T}[T]\right), \bigcup_{S} \stackrel{u}{\rightarrow} T_{T} \operatorname{Pred}_{u}(T \backslash W i n[T])\right]$.

Proof. Consider the first loop invariant. Upon entry at the while-loop the invariant is true as $\left(S, \alpha, S^{\prime}\right) \in W$ aiting for all symbolic successors of $S_{0}$. Assume that the invariant property holds after $n$ iterations the loop and let $\left(* S, \alpha, S^{\prime}\right)$ be the edge from Waiting dealt with during the the next $\left((n+1)^{\prime}\right.$ th) iteration. If $S^{\prime} \notin$ Passed before the iteration then $S^{\prime} \in$ Passed and $\left(S, \alpha, S^{\prime}\right) \in$ Depend $\left[S^{\prime}\right]$ after the iteration. Also all successors of $S^{\prime}$ are added to Waiting ensuring that the invariant holds after the iteration. If $S^{\prime} \in$ Passed before the iteration then obviously $S^{\prime} \in$ Depend $\left[S^{\prime}\right]$ after the iteration due to last statement of the reevaluate-branch.

Consider the second loop invariant. That is $\operatorname{Win}[S] \subseteq \mathcal{W}(G)$. Upon entering the while-loop this condition holds trivially. The only place at which $\operatorname{Win}[S]$ can change is in the reevaluate-branch. Now by the induction hypothesis we may assume that $W i n[T] \subseteq \mathcal{W}(G)$ whenever $S \stackrel{\alpha}{\rightarrow} T$. Then, by monotonicity of $\operatorname{Pred}_{t}$ it follows that $W_{i n^{*}} \subseteq \pi(\mathcal{W}(G)) \subseteq \mathcal{W}(G)$. Thus if the property holds before the update $\operatorname{Win}[S] \leftarrow W i n^{*}$ then it also holds after.

Consider the third loop invariant. First, in any given state of the program and for $S$ a symbolic state $\phi(S)$ will denote the value of $q \notin \operatorname{Pred}_{t}[\operatorname{Win}[S] \cup$ $\left.\bigcup_{S} \stackrel{c}{\rightarrow} \operatorname{Pred}_{c}(W i n[T]), \bigcup_{S} \stackrel{u}{\rightarrow} T \operatorname{Pred}_{u}(T \backslash W i n[T])\right]$. Upon entry of the while-loop only $S_{0} \in$ Passed and the property trivially holds as either $S_{0}$ is fully winning, as our goal set is based on locations (and hence $q \in S_{0} \backslash W i n\left[S_{0}\right]$ is impossible), or $S_{0}$ has symbolic successors which are then in the waiting list or $S_{0}$ has no symbolic successor in which case $q \notin \phi\left(S_{0}\right)$ obviously holds.

Now assume that the property holds after $n$ iterations (we will add $n$ as a superscript to indicate the value at a particular variable after the $n$ 'th iteration) and let us prove that it holds after iteration $n+1$. During this last iteration an edge $\left(S, \alpha, S^{\prime}\right)$ from Waiting $^{n}$ is processed. There are two cases to be considered depending on which branch of the while-body has been taken, i.e. either $S^{\prime} \in$ Passed $^{n}$ or $S^{\prime} \notin$ Passed $^{n}$.

Let us first consider the latter, i.e. $S^{\prime} \notin$ Passed $^{n}$. Let $q \in T \backslash$ Win $^{n+1}[T]$ for some $T \in$ Passed $^{n+1}$. If $S^{\prime}$ is not a symbolic successor of $T$ then, as $\phi(T)$ and the contents of Waiting and Depend w.r.t. $T$ are unchanged, the desired property holds after the iteration due to the induction hypothesis. If $T \stackrel{\beta}{\rightarrow} S^{\prime}$ and $T \neq S$ then by invariance property $1 .\left(T, \beta, S^{\prime}\right) \in$ Waiting $^{n}$ (as $S^{\prime} \notin$ Passed $^{n}$ 
the second options of 1 . is not an option) and hence $\left(T, \beta, S^{\prime}\right) \in$ Waiting $^{n+1}$. For the case $T=S$ and $S^{\prime} \cap\{$ Goal $\} \times \mathbb{R}_{>0}^{X}=\emptyset$, the value of $\phi(T)$ is the same before and after the iteration. Thus the induction hypothesis ensures that either $f \in$ Waiting for some edge $f=\left(T, \gamma, T^{\prime}\right)$ both before and after the iteration or $q \notin \phi(T)$ both before and after the iteration. In case $T=S$ and $S^{\prime} \cap\{$ Goal $\} \times \mathbb{R}_{\geq 0}^{X} \neq \emptyset$, the edge $\left(S, \alpha, S^{\prime}\right)=\left(T, \alpha, S^{\prime}\right)$ is being put back into the Waiting-list ensuring that the property holds.

Let us now consider the case when the processed edge $\left(S, \alpha, S^{\prime}\right)$ is of the type $S^{\prime} \in$ Passed $^{n}$. Let $q \in T \backslash$ Win $^{n+1}[T]$ for some $T \in$ Passed $^{n+1}$ :

Assume that $T \neq S$ and that $S$ is not a symbolic successor of $T$. Then by induction hypothesis the property trivially holds after the iteration. If $T \neq S$ and $T \stackrel{\beta}{\rightarrow} S$ then by the invariance property 1 . either $(T, \beta, S) \in$ Waiting $^{n}$ or $(T, \beta, S) \in$ Depend $^{n}[S]$. In the first case we satisfy the invariant property as also $(T, \beta, S) \in$ Waiting $^{n+1}$. In the second case the program ensures that $(T, \beta, S) \in$ $W_{\text {aiting }}{ }^{n+1}$ in case $W_{i n}{ }^{n+1}[S] \subsetneq W_{i n}{ }^{n}[S]$. Otherwise, $\phi(T)$ is unchanged by the iteration, and hence by the induction hypothesis either $\left(T, \gamma, T^{\prime}\right) \in$ Waiting for some $T^{\prime} \in$ Passed or $q \notin \phi(T)$ both before and after the iteration. If $T=S$ then our assumption is that $q \in S \backslash W_{i n}{ }^{n+1}[S]$. Obviously by the update of Win $[S]$ to $\phi(S)$ which has taken place during the iteration it is clear that then $q \notin \phi(S)=\phi(T)$.

Theorem 2. Upon termination of running the algorithm SOTFTR on a given timed game automaton $G$ the following holds:

1. If $q \in W$ in $[S]$ for some $S \in$ Passed then $q \in \mathcal{W}(G)$;

2. If Waiting $=\emptyset, q \in S \backslash W i n[S]$ for some $S \in$ Passed then $q \notin \mathcal{W}(G)$.

Proof. By 1. of Lemma 1 it certainly holds that if $q \in W i n[S]$ for some $S \in$ Passed then $q \in \mathcal{W}(G)$.

To show that $q \notin \mathcal{W}(G)$ in case $q \in S \backslash W i n[S]$ for some $S \in$ Passed when Waiting $=\emptyset$, we consider the following set $L$ of states based on Passed and Win upon termination:

$$
L=\{q \mid \exists S \in \text { Passed. } q \in S \backslash \operatorname{Win}[S]\}
$$

and let $M=Q \backslash L$, where $Q$ is the state-set of the timed transition system $G^{9}$. Note that $M \cap S \subseteq W i n[S]$ for all $S \in$ Passed. In fact $q \in M$ just in case whenever $q \in S$ for some $S \in$ Passed then also $q \in \operatorname{Win}[S]$.

Let $K=\{$ Goal $\} \times \mathbb{R}_{\geq 0}^{X}$. We will show that $M$ is a post-fixed point to $\lambda X . \pi(X) \cup K$ and hence that $\mathcal{W}(G) \subseteq M$. In particular, if $q \in S \backslash W$ in $[S]$ for some $S \in$ Passed then $q \notin M$ and hence $q \notin \mathcal{W}(G)$.

Now let $q \in \pi(M) \cup K$. We must prove that $q \in M$ : In case $q \in K$ clearly $q \in M$ as $S \cap K \subseteq \operatorname{Win}[S]$ for all $S \in$ Passed due to the way we initialize $\operatorname{Win}[S]$ the first time the symbolic state $S$ is encountered. Assume that $q \in \pi(M)$ and

\footnotetext{
$\overline{9}$ i.e. $Q=L \times \mathbb{R}_{\geq 0}^{X}$.
} 
that $q \in S$ for some $S \in$ Passed. We have to prove that $q \in W i n[S]$ in order to conclude that $q \in M$. Assume not, i.e. $q \notin W i n[S]$. But then according to 2 . Lemma 1 - as Waiting $=\emptyset$ - we have that:

$$
q \notin \operatorname{Pred}_{t}\left[\operatorname{Win}[S] \cup \bigcup_{S \stackrel{c}{\rightarrow} T} \operatorname{Pred}_{c}(\operatorname{Win}[T]), \bigcup_{S \stackrel{u}{\rightarrow} T} \operatorname{Pred}_{u}(T \backslash W i n[T])\right] .
$$

But since $M \cap S \subseteq$ Win $[S]$ for $S \in$ Passed it follows by monotonicity that:

$$
q \notin \operatorname{Pred}_{t}\left[(M \cap S) \cup \bigcup_{S \stackrel{c}{\rightarrow} T} \operatorname{Pred}_{c}(M \cap T), \bigcup_{S \stackrel{u}{\rightarrow} T} \operatorname{Pred}_{u}(T \backslash(M \cap T)]\right.
$$

contradicting that $q \in \pi(M)$.

Theorem 3. The following distribution law holds:

$$
\operatorname{Pred}_{t}\left(\bigcup_{i} G_{i}, \bigcup_{j} B_{j}\right)=\bigcup_{i} \bigcap_{j} \operatorname{Pred}\left(G_{i}, B\right)
$$

Proof. Assume $q \in \operatorname{Pred}_{t}\left(\bigcup_{i} G_{i}, B\right)$. Equivalently, by definition of $\left.\operatorname{Pred}_{t}, i\right) \exists \delta \in$ $\mathbb{R}_{\geq 0}$ s.t. $q \stackrel{\delta}{\rightarrow} q^{\prime}, q^{\prime} \in \bigcup_{i} G_{i}$ and $\left.i i\right) \operatorname{Post}_{[0, \delta]}(q) \subseteq \bar{B}$. $\left.i\right)$ can be written as $\exists j, \exists \delta \in$ $\mathbb{R}_{\geq 0}$ s.t. $q \stackrel{\delta}{\rightarrow} q^{\prime}, q^{\prime} \in G_{j}$, which together with $\left.i i\right)$ is equivalent to $\exists j, q \in$ $\operatorname{Pred}_{t}\left(G_{j}, B\right)$ and finally $q \in \bigcup_{i} \operatorname{Pred}_{t}\left(G_{i}, B\right)$.

Assume $q \in \operatorname{Pred}_{t}\left(G, \bigcup_{j} B_{j}\right)$. Equivalently, by definition of $\left.\operatorname{Pred}_{t}, i\right) \exists \delta \in$ $\mathbb{R}_{\geq 0}$ s.t. $q \stackrel{\delta}{\rightarrow} q^{\prime}, q^{\prime} \in G$ and $\left.i i\right) \operatorname{Post}_{[0, \delta]}(q) \subseteq \overline{\bigcup_{j} B_{j}}$. $\left.i i\right)$ can be written as $\forall \delta^{\prime} \in[0, \delta], q \stackrel{\delta^{\prime}}{\longrightarrow} \notin \bigcup_{j} B_{j}$ or $\forall j, \forall \delta^{\prime} \in[0, \delta], q \stackrel{\delta^{\prime}}{\longrightarrow} \notin B_{j}$. This is equivalent to $\forall j, \operatorname{Post}_{[0, \delta]}(q) \subseteq \overline{B_{j}}$ and together with $\left.i\right), \forall j, q \in \operatorname{Pred}_{t}\left(G, B_{j}\right)$. Finally this is equivalent $q \in \bigcap_{j} \operatorname{Pred}_{t}\left(G, B_{j}\right)$.

Theorem 4. If $B$ is a convex set, then the $\operatorname{Pred}_{t}$ operator defined in equation (1) can be expressed as:

$$
\operatorname{Pred}_{t}(G, B)=\left(G^{\swarrow} \backslash B^{\swarrow}\right) \cup\left(\left(G \cap B^{\swarrow}\right) \backslash B\right)^{\swarrow}
$$

Proof. Let us first transform the claimed expression of $\operatorname{Pred}_{t}$. It can equivalently be written as:

$$
\operatorname{Pred}_{t}(G, B)=\left(G^{\swarrow} \cap \overline{B^{\swarrow}}\right) \cup\left(G \cap B^{\swarrow} \cap \bar{B}\right)^{\swarrow}
$$

Let us first show that $\operatorname{Pred}_{t}(G, B) \subseteq\left(G^{\swarrow} \cap \overline{B^{\swarrow}}\right) \cup\left(G \cap B^{\swarrow} \cap \bar{B}\right)^{\swarrow}$

Consider $q \in \operatorname{Pred}_{t}(G, B)$. By definition of $\operatorname{Pred}_{t}$ we have $\left.i\right) \exists \delta \in \mathbb{R}_{\geq 0}$ s.t. $q \stackrel{\delta}{\rightarrow}$ $q^{\prime}, q^{\prime} \in G$ and $\left.i i\right) \operatorname{Post}_{[0, \delta]}(q) \subseteq \bar{B}$. From $i$ ), we can deduce that $q \in G^{\swarrow}$. Now if $q \notin B^{\swarrow}$, then $q \in G^{\swarrow} \cap \overline{B^{\swarrow}}$ and we have the claimed result. If $q \in B^{\swarrow}$, then by definition of the down operator, $\exists \delta^{\prime} \in \mathbb{R}_{\geq 0}$ s.t. $q \stackrel{\delta^{\prime}}{\longrightarrow} q^{\prime \prime}$ and $q^{\prime \prime} \in B$. Let $\delta^{\prime *}$ be the smallest such $\delta^{\prime}$. Similarly, let $\delta^{*}$ be the smallest $\delta$ such that $q \stackrel{\delta}{\rightarrow} q^{\prime}$, with 
$q^{\prime} \in G$ and let $q^{\prime *}$ be such that $q \stackrel{\delta^{*}}{\longrightarrow} q^{\prime *}$. From $\left.i i\right)$ we know that $\delta^{*}<\delta^{\prime *}$. So, $q^{\prime *} \notin B$ and since we have $q^{\prime} \stackrel{\delta^{\prime *}-\delta^{*}}{\longrightarrow} q^{\prime \prime *}$ with $q^{\prime \prime *} \in B, q^{\prime *} \in B^{\swarrow}$. Therefore, $q^{\prime *} \in G \cap \bar{B} \cap B^{\swarrow}$ and $q \in\left(G \cap \bar{B} \cap B^{\swarrow}\right)^{\swarrow}$, which concludes the proof of the claimed inclusion.

Now we show that $\left(G^{\swarrow} \cap \overline{B^{\swarrow}}\right) \cup\left(G \cap B^{\swarrow} \cap \bar{B}\right)^{\swarrow} \subseteq \operatorname{Pred}_{t}(G, B)$.

Assume that $q \in\left(G^{\swarrow} \cap \overline{B^{\swarrow}}\right) \cup\left(G \cap B^{\swarrow} \cap \bar{B}\right)^{\swarrow}$. First, if $q \in G^{\swarrow} \cap \overline{B^{\swarrow}}$. We have $q \in G^{\swarrow}$ and by definition of the down operator, this means that $\exists \delta \in \mathbb{R}_{\geq 0}$ s.t. $q \stackrel{\delta}{\rightarrow} q^{\prime}$ and $q^{\prime} \in G$. Furthermore, $q \in \overline{B^{\swarrow}}$ so we are done since this means that there does not exist any $\delta^{\prime} \in \mathbb{R}_{\geq 0}$ s.t. $q \stackrel{\delta^{\prime}}{\rightarrow} \in B$ or, equivalently, $\forall \delta^{\prime} \in \mathbb{R}_{\geq 0}$, $q \stackrel{\delta^{\prime}}{\rightarrow} \in \bar{B}$, which implies that $\operatorname{Post}_{[0, \delta]}(q) \subseteq \bar{B}$. If $q \in\left(G \cap B^{\swarrow} \cap \bar{B}\right)^{\swarrow}$, then by definition of the down operator, we know that $\exists \delta^{\prime} \in \mathbb{R}_{\geq 0}$ such that $q \stackrel{\delta^{\prime}}{\rightarrow} q^{\prime \prime}$ and $q^{\prime \prime} \in G \cap \bar{B} \cap B^{\swarrow}$. Let $\delta^{\prime *}$ be the smallest such $\delta^{\prime}$ and $q^{\prime \prime *}$ be such that $q \stackrel{\delta^{\prime *}}{\longrightarrow} q^{\prime \prime *}$. $q^{\prime \prime *} \in G$ so, to conclude the proof, we need only to prove that $\operatorname{Post}_{\left[0, \delta^{\prime *}\right]}(q) \subseteq \bar{B}$. Assume this is not the case, then there exists $\delta \in\left[0, \delta^{*}\right]$ s.t. $q \stackrel{\delta}{\rightarrow} \in B$. Now, on the one hand, we know that $q^{\prime \prime *} \in B^{\swarrow}$, so there exists $\delta^{\prime \prime} \in \mathbb{R}_{\geq 0}$ s.t. $q \stackrel{\delta^{\prime \prime}}{\longrightarrow} \in B$. On the other hand, $q^{\prime \prime *} \notin B$ and $\delta \leq \delta^{\prime *} \leq \delta^{\prime \prime}$. But this is not possible since $B$ is convex. So $\operatorname{Post}_{\left[0, \delta^{\prime *}\right]}(q) \subseteq \bar{B}$.

\section{B Production Cell Case Study}

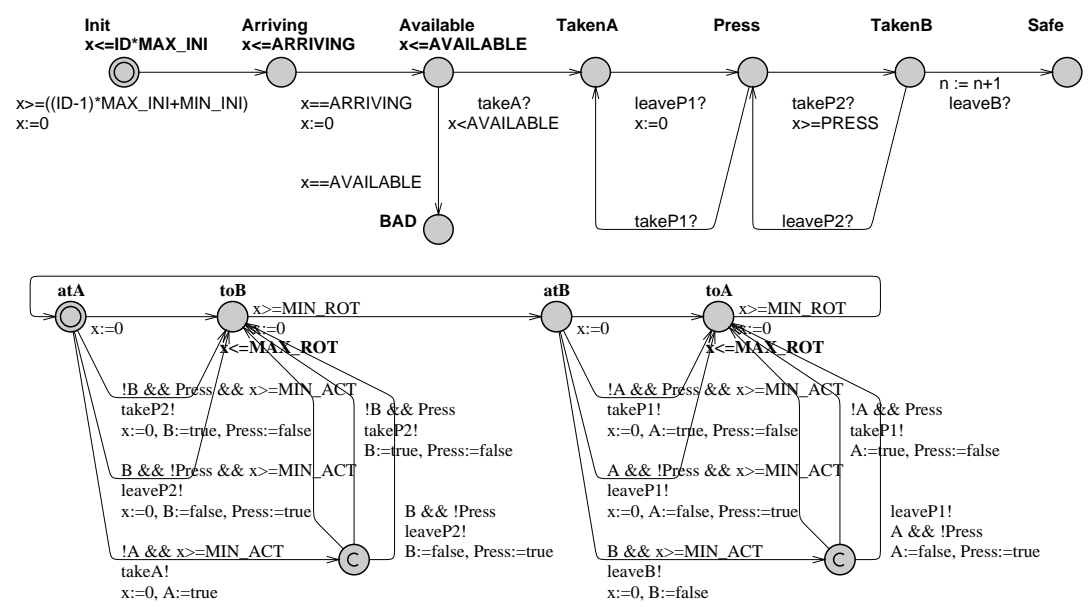

Fig. 8. UppaAL models of the plates and the robot. 


\begin{tabular}{|c|c|c|c|c|c|c|c|c|c|c|c|}
\hline \multicolumn{2}{|c|}{ Plates } & \multicolumn{2}{|c|}{ Basic } & \multicolumn{2}{|c|}{ Basic +inc } & \multicolumn{2}{|c|}{$\begin{array}{c}\text { Basic +inc } \\
\text { +pruning }\end{array}$} & \multicolumn{2}{|c|}{$\begin{array}{c}\text { Basic+lose +inc } \\
+ \text { pruning }\end{array}$} & \multicolumn{2}{|c|}{$\begin{array}{c}\text { Basic+lose +inc } \\
\text { +topt }\end{array}$} \\
\hline \multirow{3}{*}{2} & & post & pre & post & pre & post & pre & post & pre & post & pre \\
\hline & win & 1320 & 910 & 600 & 306 & 130 & 57 & 130 & 171 & 619 & 728 \\
\hline & Tose & 6514 & 4037 & 815 & 426 & 357 & 180 & 324 & 499 & $\mathrm{n} / \mathrm{a}$ & $\mathrm{n} / \mathrm{a}$ \\
\hline \multirow{2}{*}{3} & win & 57179 & 40306 & 3605 & 895 & 1678 & 325 & 1239 & 1662 & 3688 & 4332 \\
\hline & Tose & 135226 & 68595 & 5880 & 1199 & 4634 & 686 & 2862 & 3961 & $\mathrm{n} / \mathrm{a}$ & $\mathrm{n} / \mathrm{a}$ \\
\hline \multirow{2}{*}{4} & win & 3214429 & 2422067 & 17335 & 3452 & 11028 & 1257 & 6530 & 8692 & 19394 & 21937 \\
\hline & lose & 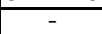 & 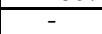 & 29376 & 5019 & 23744 & 2050 & 14059 & 18915 & $\mathrm{n} / \mathrm{a}$ & $\mathrm{n} / \mathrm{a}$ \\
\hline \multirow{2}{*}{5} & win & - & - & 76249 & 16441 & 50433 & 5855 & 22667 & 30067 & 91628 & 99492 \\
\hline & lose & - & - & 147700 & 32583 & 109727 & 11608 & 63490 & 85101 & $\mathrm{n} / \mathrm{a}$ & $\mathrm{n} / \mathrm{a}$ \\
\hline \multirow{2}{*}{6} & \begin{tabular}{|l|} 
win \\
\end{tabular} & - & - & 394952 & 94438 & 257068 & 32496 & 85563 & 113291 & 504956 & 541127 \\
\hline & Tose & - & - & 1044236 & 266660 & 730484 & \begin{tabular}{|l|}
90884 \\
\end{tabular} & 369602 & 494689 & $\mathrm{n} / \mathrm{a}$ & $\mathrm{n} / \mathrm{a}$ \\
\hline \multirow{2}{*}{7} & win & - & - & 2257872 & 560953 & 1458950 & \begin{tabular}{|l|}
192168 \\
\end{tabular} & 371037 & 491148 & 2921355 & 3111438 \\
\hline & lose & - & - & - & 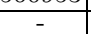 & \begin{tabular}{|l|}
5490687 \\
\end{tabular} & 715627 & \begin{tabular}{|l|}
2371542 \\
\end{tabular} & 3172594 & $\mathrm{n} / \mathrm{a}$ & $\mathrm{n} / \mathrm{a}$ \\
\hline
\end{tabular}

Table 3. Number of pre and post operations needed. '-' denotes a failed run (not enough memory). 